\title{
SUMMATION OF SLOWLY CONVERGENT SERIES
}

\author{
WALTER GAUTSCHI \\ Department of Computer Sciences, Purdue University \\ West Lafayette, Indiana 47907, U.S.A.
}

Among the applications of orthogonal polynomials described briefly on my previous visit to this Center $[9, \S 3.2]$ were slowly convergent series whose terms could be represented in terms of the Laplace transform at integer arguments. We proposed to sum such series by means of Gaussian quadrature rules applied to suitable integrals involving weight functions of Einstein and Fermi type (cf. [13]). In the meantime it transpired that the technique is applicable to a large class of numerical series and, suitably adapted, also to power series and Fourier series of interest in plate problems. In the following we give a summary of these new applications and the contexts in which they arise.

1. Spirals and series. Our first class of series may well have its roots in preArchimedean mathematics. The series arise in connection with a discrete spiral whose vertices $v_{n}$ are at a distance $\sqrt{n+1}$ from the origin,

$$
\operatorname{dist}\left(v_{n}, 0\right)=\sqrt{n+1}, \quad n=-1,0,1,2, \ldots,
$$

and at a distance 1 from one another,

$$
\operatorname{dist}\left(v_{n}, v_{n-1}\right)=1, \quad n=0,1,2, \ldots
$$

Feeling that this spiral deserves a classical name, P. J. Davis [5] has called it the discrete spiral of Theodorus, in remembrance of the 4th century B.C. mathematician Theodorus of Cyrene, reputed to have proven the irrationality of the square roots of all nonsquare integers up to 17 . (The spiral is also known as "Quadratwurzelschnecke" [14].) Davis poses the problem of "interpolating" to the discrete spiral by a smooth — indeed analytic — spiral that would enjoy the

1991 Mathematics Subject Classification: Primary 40A25; Secondary 44A10, 65D30, 33C45. Work supported in part by the National Science Foundation under grant DCR-8320561.

The paper is in final form and no version of it will be published elsewhere. 
basic distance properties (1.1), (1.2) in a continuous, rather than discrete, sense. In other words, if $v(\alpha)$ is an analytic complex-valued function representing the spiral, then, in analogy to (1.1) and (1.2), it should satisfy

$$
|v(\alpha)|=\sqrt{\alpha+1}, \quad \text { for all } \alpha>-1
$$

and

$$
|v(\alpha)-v(\alpha-1)|=1, \quad \text { for all } \alpha>0 .
$$

The problem is reminiscent of Christian Goldbach's problem posed in his 1729 letter to Euler of interpolating to the factorials. Euler's solution may well have inspired Davis to provide a solution to the spiral problem in the form of an infinite product,

$$
v(\alpha)=\prod_{k=1}^{\infty} \frac{1+i / \sqrt{k}}{1+i / \sqrt{k+\alpha}}, \quad \alpha>-1 \quad(i=\sqrt{-1})
$$

It is easily seen that this function satisfies $\left(1.1^{\prime}\right)$ and the recurrence formula

$$
v(\alpha)=\left(1+\frac{i}{\sqrt{\alpha}}\right) v(\alpha-1), \quad \alpha>0,
$$

hence also $\left(1.2^{\prime}\right)$. Furthermore, if extended to the complex plane, $v$ becomes an analytic function of $\alpha$. By simple manipulations, the spiral (1.3) can be written in polar coordinates as (cf. [5, Supplement A])

$$
\begin{gathered}
v(\alpha)=r(\alpha) \exp (i \theta(\alpha)), \\
r(\alpha)=\sqrt{1+\alpha}, \quad \theta(\alpha)=\frac{1}{2} \int_{0}^{\alpha} \sum_{k=1}^{\infty} \frac{1}{(k+\alpha)^{3 / 2}+(k+\alpha)^{1 / 2}} d \alpha .
\end{gathered}
$$

The infinite product in (1.3) and the infinite series in (1.5), while both converge, do not lend themselves to easy evaluation. It is slowly convergent series exemplified by the one in (1.5) whose summation we wish to consider first. Specifically, we will be dealing with series of the type

$$
S_{0}=\sum_{k=1}^{\infty} k^{\nu-1} r(k)
$$

and their alternating companion series

$$
S_{1}=\sum_{k=1}^{\infty}(-1)^{k-1} k^{\nu-1} r(k),
$$

where $\nu$ is a real number between 0 and 1 , and $r$ a rational function,

$$
r(s)=\frac{p(s)}{q(s)}, \quad \operatorname{deg} p \leq \operatorname{deg} q .
$$

We are ignoring, for the time being, the shift $k \mapsto k+\alpha$ typical in the series of (1.5), as well as the problem of subsequent integration. Both matters can be dealt with easily, once the necessary formulae are in place. 
2. Summation by integration. There are many ways of transforming a series into an integral. Here we use the Laplace transform,

$$
(\mathcal{L} f)(s)=\int_{0}^{\infty} e^{-s t} f(t) d t
$$

assuming that the general term, $a_{k}$, of the series can be represented as the Laplace transform of some function, $f$, evaluated at the integer $k$,

$$
a_{k}=(\mathcal{L} f)(k), \quad k=1,2,3, \ldots
$$

One then gets

$$
\sum_{k=1}^{\infty} a_{k}=\sum_{k=1}^{\infty} \int_{0}^{\infty} e^{-k t} f(t) d t=\int_{0}^{\infty} f(t) e^{-t} \sum_{k=1}^{\infty} e^{-(k-1) t} d t=\int_{0}^{\infty} \frac{f(t)}{t} \cdot \frac{t}{e^{t}-1} d t
$$

The second factor in the last integral is known, in solid state physics, as Einstein's function,

$$
\varepsilon(t)=\frac{t}{e^{t}-1} .
$$

(It is, of course, also the generating function of the Bernoulli numbers.) Thus,

$$
\sum_{k=1}^{\infty} a_{k}=\int_{0}^{\infty} \frac{f(t)}{t} \varepsilon(t) d t, \quad a_{k}=(\mathcal{L} f)(k)
$$

Entirely analogously, one finds for alternating series

$$
\sum_{k=1}^{\infty}(-1)^{k-1} a_{k}=\int_{0}^{\infty} f(t) \varphi(t) d t, \quad a_{k}=(\mathcal{L} f)(k),
$$

where

$$
\varphi(t)=\frac{1}{e^{t}+1}
$$

is known as Fermi's function. Since both functions $\varepsilon$ and $\varphi$ have strings of poles along the imaginary axis, it will be convenient to treat them as weight functions and to deal with the respective integrals in (2.4), (2.5) by means of weighted Gaussian quadrature. This is basically the approach we are going to take to evaluate the series $S_{0}, S_{1}$ in (1.6) and (1.7), respectively. The main problem is to identify the function $f$ in $(2.2)$, which requires a little bit of special functions.

3. Special function theory. By a preliminary use of partial fraction expansion, attention may be restricted to rational functions of the form

$$
r(s)=\frac{1}{(s+a)^{m}}, \quad \operatorname{Re} a \geq 0, \operatorname{Im} a \geq 0, m \geq 1,
$$


where the condition Re $a \geq 0$ can be achieved, if necessary, by first summing a few of the initial terms directly. We thus consider

$$
S_{0}=\sum_{k=1}^{\infty} \frac{k^{\nu-1}}{(k+a)^{m}}, \quad S_{1}=\sum_{k=1}^{\infty}(-1)^{k-1} \frac{k^{\nu-1}}{(k+a)^{m}} .
$$

Since both $k^{\nu-1}$ and $(k+a)^{-m}$ are Laplace transforms of power functions (multiplied by an exponential in the latter case), we can use the convolution theorem to find the function $f$ in the representation (2.2) for

$$
a_{k}=k^{\nu-1} \cdot(k+a)^{-m} .
$$

One finds, after a change of variables,

$$
f(t)=\frac{t^{m-\nu} e^{-a t}}{(m-1) ! \Gamma(1-\nu)} \int_{0}^{1} e^{a t u}(1-u)^{m-1} u^{-\nu} d u
$$

The integral on the right relates the function $f$ to a Kummer function $M(\alpha, \beta, z)$ with parameters $\alpha=1-\nu, \beta=m+1-\nu$ and variable $z=a t$ (cf. [1, Eq. 13.2.1]),

$$
f(t)=t^{1-\nu} g_{m-1}(t ; a, \nu),
$$

where

$$
g_{n}(t ; a, \nu)=g_{n}(t)=\frac{t^{n} e^{-a t}}{\Gamma(n+2-\nu)} M(1-\nu, n+2-\nu, a t), \quad n=0,1,2, \ldots
$$

The well-known recurrence relation relative to the second parameter in Kummer's function [1, Eq. 13.4.2] then yields

$$
\begin{aligned}
g_{n+1}(t) & =\frac{1}{n+1}\left\{\left(t+\frac{n+1-\nu}{a}\right) g_{n}(t)-\frac{t}{a} g_{n-1}(t)\right\}, \quad n=0,1,2, \ldots, \\
g_{-1}(t) & =\frac{t^{-1}}{\Gamma(1-\nu)} .
\end{aligned}
$$

(It is assumed here that $a \neq 0$; similar, even simpler, formulae hold for $a=0$.) The only function we need to identify, therefore, is $g_{0}(t)$; it can be expressed $[1$, Eq. 13.6.10] as

$$
g_{0}(t ; a, \nu)=e^{-a t} \gamma^{*}(1-\nu,-a t)
$$

in terms of Tricomi's form of the incomplete gamma function [1, Eq. 6.5.4]

$$
\gamma^{*}(\lambda, z)=\frac{z^{-\lambda}}{\Gamma(\lambda)} \int_{0}^{z} e^{-t} t^{\lambda-1} d t
$$

an entire function in both its variables. The functions $g_{n}(t), n=0,1,2, \ldots$, therefore, are all entire functions of $t$. 
Of particular interest to us is the parameter value $\nu=1 / 2$ (cf. (1.5)), for which Tricomi's incomplete gamma function becomes Dawson's integral [1, Eqs. 6.5.18, 7.1.17],

$$
F(z)=e^{-z^{2}} \int_{0}^{z} e^{t^{2}} d t
$$

and in terms of which

$$
g_{0}\left(t ; a, \frac{1}{2}\right)=\frac{2}{\sqrt{\pi}} \frac{F(\sqrt{a t})}{\sqrt{a t}} .
$$

The presence of the square roots on the right gives only the appearance of singular behavior; in reality, they cancel out, since $F$ is an odd function.

4. Summation formulae. We now combine the results of $\S \S 2,3$. The relation (2.4), together with (3.3) and (3.4), yields

$$
\sum_{k=1}^{\infty} \frac{k^{\nu-1}}{(k+a)^{m}}=\int_{0}^{\infty} t^{-\nu} \varepsilon(t) \cdot g_{m-1}(t ; a, \nu) d t
$$

where $\varepsilon$ is given by (2.3) and the function $g_{m-1}$ is computable via (3.6) and (3.7) (resp. (3.10) if $\nu=1 / 2$ ). Likewise, (2.5) and (3.3), (3.4) give

$$
\sum_{k=1}^{\infty}(-1)^{k-1} \frac{k^{\nu-1}}{(k+a)^{m}}=\int_{0}^{\infty} t^{-\nu} \varphi(t) \cdot t g_{m-1}(t ; a, \nu) d t
$$

with $\varphi$ defined in (2.6). Since $g_{m-1}$ is a smooth function of $t$ - indeed an entire function - it makes sense to evaluate the integrals in (4.1) and (4.2) by Gaussian quadrature relative to the weight functions $t^{-\nu} \varepsilon(t)$ and $t^{-\nu} \varphi(t)$, respectively. Although these are "nonclassical" weight functions, and the corresponding orthogonal polynomials therefore unknown, the required Gaussian quadrature rules can be generated numerically by known methods (cf. [10]). For $\nu=1 / 2$, auxiliary tables of recursion coefficients for the respective orthogonal polynomials can be found in the Appendix to [11].

It is now easy to incorporate a shift in the summation index of the series (4.1), (4.2). Indeed, a well-known property of the Laplace transform tells us that to a translation $k \mapsto k+b$ in the variable $k$ there corresponds a multiplication by an exponential, $f(t) \mapsto e^{-b t} f(t)$, in the function $f$. Therefore, if we denote $b+a$ in the denominator of the "shifted" series (4.1), (4.2) again by $a$, and adjust the second parameter in $g_{m-1}$ accordingly, we obtain

$$
\begin{aligned}
\sum_{k=1}^{\infty} \frac{(k+b)^{\nu-1}}{(k+a)^{m}} & =\int_{0}^{\infty} t^{-\nu} \varepsilon(t) \cdot e^{-b t} g_{m-1}(t ; a-b, \nu) d t \\
\sum_{k=1}^{\infty}(-1)^{k-1} \frac{(k+b)^{\nu-1}}{(k+a)^{m}} & =\int_{0}^{\infty} t^{-\nu} \varphi(t) \cdot t e^{-b t} g_{m-1}(t ; a-b, \nu) d t .
\end{aligned}
$$


The first of these series, when $\nu=1 / 2$ and $a=\alpha+1, b=\alpha, m=1$, becomes the series in (1.5), so that

$$
\begin{aligned}
\sum_{k=1}^{\infty} \frac{1}{(k+\alpha)^{3 / 2}+(k+\alpha)^{1 / 2}} & =\int_{0}^{\infty} t^{-1 / 2} \varepsilon(t) \cdot e^{-\alpha t} g_{0}\left(t ; 1, \frac{1}{2}\right) d t \\
& =\frac{2}{\sqrt{\pi}} \int_{0}^{\infty} t^{-1 / 2} \varepsilon(t) e^{-\alpha t} \frac{F(\sqrt{t})}{\sqrt{t}} d t
\end{aligned}
$$

where (3.10) has been used to get the last expression. Integration with respect to $\alpha$, as required in (1.5), is now easily accomplished by integrating under the integral sign in (4.3),

$$
\int_{0}^{\alpha} \sum_{k=1}^{\infty} \frac{1}{(k+\alpha)^{3 / 2}+(k+\alpha)^{1 / 2}} d \alpha=\frac{2 \alpha}{\sqrt{\pi}} \int_{0}^{\infty} t^{-1 / 2} \varepsilon(t) \frac{1-e^{-\alpha t}}{\alpha t} \frac{F(\sqrt{t})}{\sqrt{t}} d t
$$

The integral on the right is equally amenable to Gaussian quadrature as all the integrals before, requiring only a little extra care in evaluating the third factor in the integrand when $\alpha t$ is small.

\section{Examples}

EXAMPLE 5.1. $S_{0}=\sum_{k=1}^{\infty} k^{-1 / 2} /(k+a)^{m}, a>0$. For this series we apply Gauss quadrature with weight function $t^{-1 / 2} \varepsilon(t)$ to the integral on the right of (4.1) (where $\nu=1 / 2$ ) and use (3.10) together with (3.6) to evaluate $g_{m-1}$. Dawson's integral $F$ in (3.10) can be computed to up to 20 decimal places with the help of known rational approximations [4]. It is found, for $m=1$, that 20 correct decimal digits can be obtained, when $a=.5,1 ., 2$., if one applies $n$-point quadrature with respectively $n=15,25,35$. As $a$ increases, convergence slows down; with $n=40$, for example, one gets only about 12 correct digits for $a=4$., and 7 for $a=8$. Similar results are observed for $m>1$.

The slowdown of convergence with increasing $a$ has its reason in the behavior of the function $g_{0}(t ; a, 1 / 2)$ in (3.10), which as $a \rightarrow \infty$ approaches the discontinuous function equal to $2 / \sqrt{\pi}$ at $t=0$, and 0 for $t>0$. It is possible, however, to regain fast convergence, even for large values of $a$, if the series is "layered" as follows. Let $a_{0}=\lfloor a\rfloor$ be the largest integer $\leq a$, so that $a=a_{0}+a_{1}$, where $0 \leq a_{1}<1$. Then put $k=\lambda+\kappa a_{0}$ and carry out the summation by summing over all $\kappa=0,1,2, \ldots$ for each $\lambda=1,2, \ldots, a_{0}$. Thus,

$$
\begin{aligned}
S_{0} & =\sum_{\lambda=1}^{a_{0}} \sum_{\kappa=0}^{\infty} \frac{\left(\lambda+\kappa a_{0}\right)^{-1 / 2}}{\left(\lambda+\kappa a_{0}+a_{0}+a_{1}\right)^{m}} \\
& =a_{0}^{-(m+1 / 2)} \sum_{\lambda=1}^{a_{0}}\left\{\sum_{\kappa=1}^{\infty} \frac{\left(\kappa+\lambda / a_{0}\right)^{-1 / 2}}{\left(\kappa+1+\left(\lambda+a_{1}\right) / a_{0}\right)^{m}}+\frac{\left(\lambda / a_{0}\right)^{-1 / 2}}{\left(1+\left(\lambda+a_{1}\right) / a_{0}\right)^{m}}\right\} .
\end{aligned}
$$

To the inner series we can now apply $\left(4.1^{\prime}\right)$ with the effective parameter in $g_{m-1}$ being $a-b=1+\left(\lambda+a_{1}\right) / a_{0}-\lambda / a_{0}=1+a_{1} / a_{0} \approx 1$, and the parameter in the 
exponential, $b=\lambda / a_{0} \leq 1$. Gauss quadrature now converges rapidly for each $\lambda$, and it only remains to execute the finite summation over $\lambda$.

EXAMPLE 5.2. $S_{1}=\sum_{k=1}^{\infty}(-1)^{k-1} k^{-1 / 2} /(k+i \alpha), \alpha>0$. Here, $a=i \alpha, \nu=\frac{1}{2}$ and $m=1$ in (4.2), and a simple computation gives

$$
g_{0}\left(t ; i \alpha, \frac{1}{2}\right)=\sqrt{\frac{2}{\alpha t}} e^{-i \alpha^{2} t^{2}}\left[C\left(\sqrt{\frac{2 \alpha t}{\pi}}\right)+i S\left(\sqrt{\frac{2 \alpha t}{\pi}}\right)\right],
$$

where $C, S$ are the Fresnel integrals. They, too, can be computed to high accuracy from rational approximations [3]. As is to be expected, Gauss quadrature in (4.2), with weight function $t^{-1 / 2} \varphi(t)$, converges rapidly for $\alpha$ relatively small, but has difficulty in converging when $\alpha$ becomes large. A device of stratified summation, similar to the one in (5.1), however, is again applicable to restore fast convergence for large $\alpha$ (for details, see [11]).

It should be pointed out that convergence acceleration algorithms, such as the $\varepsilon$-algorithm, can be applied successfully to the series $S_{1}$, but are ineffective in the case of the series $S_{0}$. An explanation for this [7] can be given on the basis of Theorem 1 in [15, p. 127].

6. Series occurring in the theory of plates. The mathematical treatment of contact problems for laterally loaded, unilaterally constrained square plates has recently led to another class of series [6],

$$
R_{p}(z)=\sum_{k=0}^{\infty} \frac{z^{2 k+1}}{(2 k+1)^{p}}, \quad z \in \mathbb{C},|z| \leq 1, p=2 \text { or } 3,
$$

which are also slowly convergent when $|z|$ is close, or equal, to 1 . Since $R_{p}(-z)=$ $-R_{p}(z)$ and $R_{p}(\bar{z})=\overline{R_{p}(z)}$, it suffices to consider the case where $z$ is in the first quadrant of the complex plane. The corresponding alternating series,

$$
S_{p}(z)=\sum_{k=0}^{\infty}(-1)^{k} \frac{z^{2 k+1}}{(2 k+1)^{p}}, \quad z \in \mathbb{C},|z| \leq 1, p=2 \text { or } 3,
$$

no longer gives anything new, since clearly

$$
S_{p}(z)=i R_{p}(-i z) .
$$

Values of $z$ that are of particular interest in applications are

$$
z=A, 0<A \leq 1 \text { and } z=e^{i \alpha}, \alpha \in \mathbb{R} .
$$

To the series in (6.1) we can again apply the technique of $\S 2$, with the slight twist, however, that only the coefficient of the general term is expressed as a Laplace transform, and the power of the variable is left as is. In other words, we write

$$
\frac{1}{(k+1 / 2)^{p}}=(\mathcal{L} f)(k), \quad f(t)=\frac{1}{(p-1) !} t^{p-1} e^{-t / 2}
$$


and transform $R_{p}$ as follows:

$$
\begin{aligned}
R_{p}(z) & =\frac{z}{2^{p}} \sum_{k=0}^{\infty} \frac{z^{2 k}}{(k+1 / 2)^{p}}=\frac{z}{2^{p}} \sum_{k=0}^{\infty} z^{2 k} \int_{0}^{\infty} e^{-k t} \cdot \frac{t^{p-1} e^{-t / 2}}{(p-1) !} d t \\
& =\frac{z}{2^{p}(p-1) !} \int_{0}^{\infty} \sum_{k=0}^{\infty}\left(z^{2} e^{-t}\right)^{k} \cdot t^{p-1} e^{-t / 2} d t \\
& =\frac{z}{2^{p}(p-1) !} \int_{0}^{\infty} \frac{1}{1-z^{2} e^{-t}} t^{p-1} e^{-t / 2} d t,
\end{aligned}
$$

that is,

$$
R_{p}(z)=\frac{z}{2^{p}(p-1) !} \int_{0}^{\infty} \frac{t^{p-1} e^{t / 2}}{e^{t}-z^{2}} d t .
$$

In the case $z=1$, there again emerges Einstein's function $\varepsilon(t)$ (cf. (2.3)), since

$$
R_{p}(1)=\frac{1}{2^{p}(p-1) !} \int_{0}^{\infty} \varepsilon(t) \cdot t^{p-2} e^{t / 2} d t
$$

It is simpler, however, to make use of the known relationship with the Riemann zeta function,

$$
R_{p}(1)=\left(1-2^{-p}\right) \zeta(p) .
$$

More interesting is the case $z \neq 1$. The integral in (6.5), in this case, can no longer be easily evaluated numerically, when $|z|$ is close to 1 , even if the weight function $\varepsilon(t)$ is pulled out, because a steep boundary layer near $t=0$ prevents numerical integration from being effective. We instead make the change of variables $e^{-t} \mapsto t$, and thus write $R_{p}$ in the form

$$
R_{p}(z)=\frac{1}{2^{p}(p-1) ! z} \int_{0}^{1} \frac{w_{p}(t)}{z^{-2}-t} d t,
$$

where

$$
w_{p}(t)=\frac{[\ln (1 / t)]^{p-1}}{\sqrt{t}}, \quad 0<t \leq 1 .
$$

This expresses $R_{p}(z)$ as a Stieltjes transform (evaluated at $z^{-2}$ ) of the weight function $w_{p}$ in (6.8). For $S_{p}(z)$ in (6.2), the analogous result follows by using (6.7) in (6.3),

$$
S_{p}(z)=\frac{1}{2^{p}(p-1) ! z} \int_{0}^{1} \frac{w_{p}(t)}{z^{-2}+t} d t .
$$

In effect, $-S_{p}(z)$ is the Stieltjes transform of $w_{p}$ evaluated at $-z^{-2}$. Since under the assumption $|z| \leq 1, z \neq 1$, both $z^{-2}$ and $-z^{-2}$ lie outside the interval $[0,1]$ on which $w_{p}$ is supported, we can avail ourselves of powerful methods for evaluating 
the Stieltjes transform outside the support interval. These will be described in the next section. Before doing so, however, we mention an alternative method of computing $R_{p}(z)$.

Boersma and Dempsey [2] recently observed that $R_{p}(z)$ in (6.1) is Legendre's chi-function of order $p$ and can be expanded in powers of $\log z$. Specifically, for $p=2$, they obtain

$$
\begin{aligned}
R_{2}(z)= & \frac{\pi^{2}}{8}+\sum_{k=0}^{\infty} \frac{1-2^{-(2 k+1)}}{k+1}\left(2^{2 k} B_{2 k+2}\right) \frac{(\log z)^{2 k+3}}{(2 k+3) !} \\
& +\frac{1}{2} \log z[1+\log 2-\log (-\log z)],
\end{aligned}
$$

where $B_{2 k+2}$ are the Bernoulli numbers. The series in (6.10) converges uniformly for $|\log z| \leq \pi$, since

$$
2^{2 k} B_{2 k+2}=O\left(\frac{(2 k+2) !}{\pi^{2 k+2}}\right), \quad k \rightarrow \infty .
$$

In particular, we have convergence for the first choice of $z$ in (6.4) if

$$
e^{-\pi} \leq A \leq 1 \text {. }
$$

For the second choice, $z=e^{i \alpha}$, the real and imaginary parts of (6.10) yield, for $|\alpha| \leq \pi$

$$
\begin{aligned}
\operatorname{Re} R_{2}\left(e^{i \alpha}\right)= & \frac{\pi^{2}}{8}-\frac{\pi}{4}|\alpha|, \\
\operatorname{Im} R_{2}\left(e^{i \alpha}\right)= & -\sum_{k=0}^{\infty}(-1)^{k} \frac{1-2^{-(2 k+1)}}{k+1}\left(2^{2 k} B_{2 k+2}\right) \frac{\alpha^{2 k+3}}{(2 k+3) !} \\
& +\frac{1}{2} \alpha(1+\log 2-\log |\alpha|) .
\end{aligned}
$$

These expansions are particularly useful in the immediate neighborhood of $z=1$ (cf. $§ 8$ ). Similar expansions hold for $p=3$, and indeed for arbitrary integers $p \geq 2$ (cf. [2, Eqs. (2.4), (3.2)]).

7. Backward recurrence algorithm for Stieltjes transforms. Let $w$ be a positive weight function on $[0,1]$ (such as $w=w_{p}$ in $(6.8)$ ) and $\pi_{n}(\cdot ; w)$ be the (monic) $n$ th-degree orthogonal polynomial relative to the weight function $w$. It is well known that there exist constants $\alpha_{k}=\alpha_{k}(w) \in \mathbb{R}, \beta_{k}=\beta_{k}(w)>0$, depending on the weight function $w$, such that $\left\{\pi_{k}(z ; w)\right\}$ is a solution of the three-term recurrence relation

$$
y_{k+1}=\left(z-\alpha_{k}\right) y_{k}-\beta_{k} y_{k-1}, \quad k=0,1,2, \ldots,
$$

with initial values $y_{0}=1, y_{-1}=0$. Another solution is known to be

$$
\varrho_{-1}(z)=1, \quad \varrho_{k}(z)=\int_{0}^{1} \frac{\pi_{k}(t ; w)}{z-t} w(t) d t, \quad k=0,1,2, \ldots
$$


This indeed is a minimal solution of $(7.1)$ if $z$ is outside the interval $[0,1]$, i.e. (cf. $[8])$,

$$
\lim _{k \rightarrow \infty} \frac{\varrho_{k}(z)}{y_{k}}=0, \quad z \in \mathbb{C} \backslash[0,1],
$$

for any solution $\left\{y_{k}\right\}$ linearly independent of $\left\{\varrho_{k}(z)\right\}$. As minimal solution, the $\varrho_{k}$ are uniquely determined by one starting value, for example, $\varrho_{-1}=1$. Indeed, to compute $\varrho_{n}(z)$ for $0 \leq n \leq N$, one has the following algorithm [8]: For any $\nu>N$, let

$$
r_{\nu}^{[\nu]}(z)=0, \quad r_{n-1}^{[\nu]}(z)=\frac{\beta_{n}}{z-\alpha_{n}-r_{n}^{[\nu]}(z)}, \quad n=\nu, \nu-1, \ldots, 0,
$$

where $\alpha_{n}, \beta_{n}$ are the coefficients in (7.1). Define

$$
\varrho_{-1}^{[\nu]}(z)=1, \quad \varrho_{n}^{[\nu]}(z)=r_{n-1}^{[\nu]}(z) \varrho_{n-1}^{[\nu]}(z), \quad n=0,1, \ldots, N .
$$

Then

$$
\lim _{\nu \rightarrow \infty} \varrho_{n}^{[\nu]}(z)=\varrho_{n}(z), \quad n=0,1, \ldots, N, z \in \mathbb{C} \backslash[0,1] .
$$

Now observe that

$$
\varrho_{0}(z)=\int_{0}^{1} \frac{w(t)}{z-t} d t
$$

is the Stieltjes transform of $w$. It can therefore be computed by putting $N=0$ in (7.5), (7.6), i.e.

$$
\varrho_{0}(z)=r_{-1}^{[\infty]}(z)=\lim _{\nu \rightarrow \infty} r_{-1}^{[\nu]}(z)
$$

where $r_{-1}^{[\nu]}(z)$, for any $\nu$, is obtained by backward recursion as in (7.4). The procedure is readily applied to $R_{p}(z)$ in $(6.7)$ and $S_{p}(z)$ in (6.9). The argument $z$, then, has to be replaced by $z^{-2}$ and $-z^{-2}$, respectively. For the $z$-values in $(6.4)$ of interest in applications, practical guidelines have been developed for choosing $\nu$ in such a way that $r_{-1}^{[\nu]}\left(z^{-2}\right)$ sufficiently approximates $r_{-1}^{[\infty]}\left(z^{-2}\right)($ cf. $[12, \S 4])$.

8. Numerical considerations. In the important case where $z=A, 0<$ $A \leq 1$ (cf. (6.4)), neither the backward recurrence algorithm (7.4), (7.8) applied to (6.7), nor the series expansion (6.10) and similar expansions for $p>2$, allow us to evaluate $R_{p}(z)$ in the entire range of interest. The former method fails in the immediate neighborhood of $A=1$, the latter when $A<e^{-\pi}$ (cf. (6.11)). Combining both methods, however, has the potential of yielding an effective procedure applicable over the whole interval $0<A \leq 1$, especially if a suitable separation point $A_{0}$ can be found such that backward recurrence is advantageous for $0<A \leq A_{0}$, and series expansion for $A_{0}<A \leq 1$. The experimental determination of such an $A_{0}$ is the subject of this section. 
We base our determination of the separation point $A_{0}$ on the computational work required to evaluate $R_{p}(z)$ to a prescribed accuracy. More precisely, in the case of the backward recurrence algorithm, for given $\varepsilon>0$ and $z$, we first determine the smallest integer $\nu$ such that $\left|r_{-1}^{[\nu]}\left(z^{-2}\right)-r_{-1}^{[\nu-1]}\left(z^{-2}\right)\right| \leq \varepsilon\left|r_{-1}^{[\nu]}\left(z^{-2}\right)\right|$, and then count the number of arithmetic operations required to evaluate $R_{p}(z) \approx$ $r_{-1}^{[\nu]}\left(z^{-2}\right) /\left(2^{p}(p-1) ! z\right)$ (cf. (6.7)) for this particular $\nu$. We do the same for the series in (6.10) (and the analogous series for $p=3$ ), determining the smallest $n$ such that $\left|s_{n}(z)-s_{n-1}(z)\right| \leq \varepsilon\left|s_{n}(z)\right|$, where $s_{n}(z)$ is the partial sum from $k=0$ to $k=n$. We consider one method preferable over the other if the computational work so determined is less.

It is seen from (7.4) and (6.7) that backward recursion (with prescribed $\nu$ ) requires $3 \nu+6$ operations (additions + multiplications). The $n$th partial sum $s_{n}(z)$ for the series in (6.10), assuming that all required coefficients are precomputed and Horner's scheme is used to evaluate the sum, involves $2 n+8$ operations plus 2 logarithms. For the analogous series with $p=3$, the count is $2 n+10$ plus 2 logarithms.

For selected values of $A$, Table 8.1 illustrates the work required to obtain three accuracy levels: relative errors $\varepsilon$ of $.5 \times 10^{-12}, .5 \times 10^{-16}$ and $.5 \times 10^{-20}$. The smallest integers $\nu$ and $n$ (in the above discussion) achieving these accuracies are shown in the columns headed " $\nu$ " and " $n$ ", respectively. Next to them are shown the corresponding number of arithmetic operations, where one logarithm is assessed respectively 14, 16, 20 operations for the above three accuracy levels (corresponding to standard rational approximations). It can be seen that for both $p=2$ and $p=3$, backward recursion is about equally expensive as series expansion when $A=\exp (-.05 \pi)$, more expensive for larger values of $A$, and less expensive

Table 8.1. Computational work: backward recursion vs. series expansion

\begin{tabular}{|c|rrrr|rrrr|}
\hline \multirow{2}{*}{$A$} & \multicolumn{5}{|c|}{$p=2$} & \multicolumn{4}{c|}{$p=3$} \\
\cline { 2 - 8 }$e^{-.01 \pi}$ & $\nu$ & $o p$ & $n$ & $o p$ & $\nu$ & $o p$ & $n$ & $o p$ \\
& 25 & 81 & 2 & 40 & 21 & 69 & 1 & 40 \\
$e^{-.03 \pi}$ & 34 & 108 & 3 & 46 & 30 & 96 & 2 & 46 \\
& 43 & 135 & 4 & 56 & 39 & 123 & 3 & 56 \\
& 15 & 51 & 3 & 42 & 13 & 45 & 2 & 42 \\
$e^{-.05 \pi}$ & 20 & 66 & 4 & 48 & 19 & 63 & 3 & 48 \\
& 26 & 84 & 5 & 58 & 24 & 78 & 4 & 58 \\
& 12 & 42 & 3 & 42 & 11 & 39 & 3 & 44 \\
$e^{-.1 \pi}$ & 16 & 54 & 5 & 50 & 15 & 51 & 4 & 50 \\
& 20 & 66 & 6 & 60 & 19 & 63 & 5 & 60 \\
& 9 & 33 & 4 & 44 & 8 & 30 & 4 & 46 \\
$e^{-.5 \pi}$ & 11 & 39 & 6 & 52 & 11 & 39 & 6 & 54 \\
& 14 & 48 & 8 & 64 & 13 & 45 & 7 & 64 \\
& 4 & 18 & 16 & 68 & 3 & 15 & 14 & 66 \\
& 5 & 21 & 22 & 84 & 4 & 18 & 20 & 82 \\
& 6 & 24 & 26 & 100 & 5 & 21 & 25 & 100 \\
\hline
\end{tabular}


for smaller values of $A$, by a factor of about $4-5$ when $A=\exp (-.5 \pi)$. Thus, the switchover point is $A_{0} \approx \exp (-.05 \pi)=.855$.

For $z=e^{i \alpha}, 0 \leq \alpha \leq \pi / 2$, the partial sums of the series (6.12) and the analogous series for $p=3$ (again summed by Horner's scheme with precomputed coefficients) turn out to be more efficient, even away from $\alpha=0$, than backward recursion (7.4), since the latter involves complex arithmetic.

\section{References}

[1] M. Abramowitz and I. A. Stegun (eds.), Handbook of Mathematical Functions, NBS Appl. Math. Ser. 55, U.S. Government Printing Office, Washington, D.C., 1964.

[2] J. Boersma and J. P. Dempsey, On the numerical evaluation of Legendre's chi-function, Math. Comp. 59 (1992), 157-163.

[3] W. J. Cody, Chebyshev approximations for the Fresnel integrals, Math. Comp. 22 (1968), 450-453. Loose microfiche suppl. A1-B4.

[4] W. J. Cody, K. A. Paciorek and H. C. Thacher, Jr., Chebyshev approximations for Dawson's integral, ibid. 24 (1970), 171-178.

[5] P. J. Davis, Spirals: from Theodorus to Chaos, AK Peters, Boston 1993.

[6] K. M. Dempsey, D. Liu and J. P. Dempsey, Plana's summation formula for $\sum_{m=1,3, \ldots}^{\infty} m^{-2} \sin (m \alpha), m^{-3} \cos (m \alpha), m^{-2} A^{m}, m^{-3} A^{m}$, Math. Comp. 55 (1990), 693-703.

[7] B. Gabutti, personal communication, June 1991.

[8] W. Gautschi, Minimal solutions of three-term recurrence relations and orthogonal polynomials, Math. Comp. 36 (1981), 547-554.

[9] - Some applications and numerical methods for orthogonal polynomials, in: Numerical Analysis and Mathematical Modelling, A. Wakulicz (ed.), Banach Center Publ. 24, PWNPolish Scientific Publishers, Warszawa 1990, 7-19.

[10] - Computational aspects of orthogonal polynomials, in: Orthogonal Polynomials - Theory and Practice, P. Nevai (ed.), NATO Adv. Sci. Inst. Ser. C: Math. Phys. Sci. 294, Kluwer, Dordrecht 1990, 181-216.

[11] -, A class of slowly convergent series and their summation by Gaussian quadrature, Math. Comp. 57 (1991), 309-324.

[12] - On certain slowly convergent series occurring in plate contact problems, ibid. 57 (1991), 325-338.

[13] W. Gautschi and G. V. Milovanović, Gaussian quadrature involving Einstein and Fermi functions with an application to summation of series, ibid. 44 (1985), 177-190.

[14] E. Hlawka, Gleichverteilung und Quadratwurzelschnecke, Monatsh. Math. 89 (1980), $19-44$.

[15] J. Wimp, Sequence Transformations and Their Application, Math. Sci. Engrg. 154, Academic Press, New York 1981. 